\title{
Ehrlichia and Anaplasma ${ }^{1}$
}

\author{
Yuexun Tian, Cynthia C. Lord, and Eva A. Buckner²
}

\section{Synopsis}

Ehrlichia and Anaplasma are bacteria that cause diseases, known as ehrlichiosis and anaplasmosis, in humans and other animals. Ehrlichia and Anaplasma are primarily transmitted through the bites of infected hard ticks, such as the lone star tick, the blacklegged tick, and the American dog tick. In the United States, four Ehrlichia species and a single Anaplasma species are known to cause disease in humans (Ehrlichia chaffeensis, Ehrlichia ewingii, Ehrlichia muris eauclairensis, Panola Mountain Ehrlichia and Anaplasma phagocytophilum). However, human ehrlichiosis and anaplasmosis have not been a severe problem in Florida with no more than 40 confirmed cases every year for each disease. In addition to humans, multiple Ehrlichia and Anaplasma species cause disease in domestic animals and livestock, resulting in veterinary costs and potentially significant economic losses. Heartwater in domestic ruminants and canine ehrlichiosis in dogs are two significant diseases in animals caused by Ehrlichia. Tick prevention and management are the best ways to reduce ehrlichiosis and anaplasmosis in humans and animals.

\section{Introduction}

Ehrlichia (Figure 1a) and Anaplasma (Figure 1b) are related bacterial genera in the Order Rickettsiales, Family Anaplasmataceae. Species in Ehrlichia and Anaplasma are obligate intracellular bacteria that are responsible for diseases known as ehrlichiosis and anaplasmosis, respectively, in humans and other animals. Because of the lack of specific symptoms, diagnostic of these two species relies on serological and/or molecular techniques.

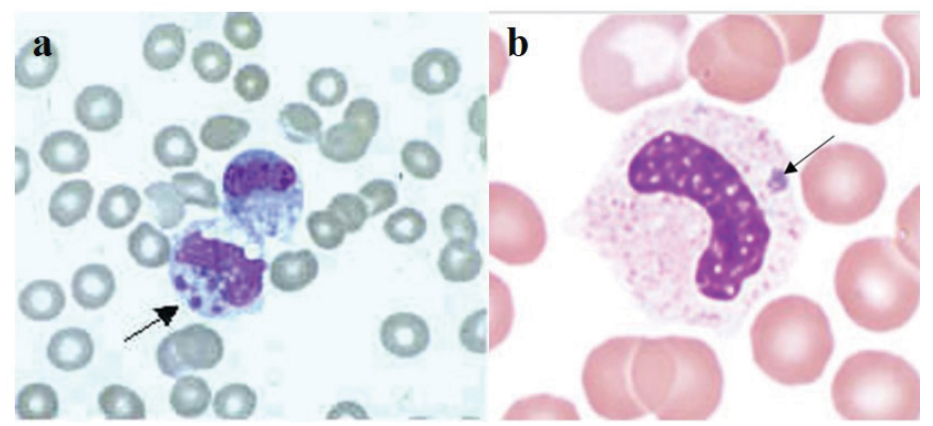

Figure 1. (a) Stained blood showing Ehrlichia chaffeensis (arrow) in a white blood cell. (b) Stained blood smear showing Anaplasma phagocytophilum (arrow) in a white blood cell.

Credits: (a) Centers for Disease Control and Prevention; (b) Bobbi S. Pritt, Mayo Clinic

Hard ticks (Figure 2), such as Amblyomma americanum Linnaeus, the lone star tick; Ixodes scapularis Say, the blacklegged tick; and Dermacentor variabilis Say, the American dog tick, are the primary biological vectors of both Ehrlichia and Anaplasma bacteria to humans and other animals. The majority of these bacteria are biologically transmitted (with pathogen reproduction within the host) to human through the bite of infected ticks. In ticks, certain Ehrlichia and Anaplasma bacterial species can be transmitted through transstadial transmission (from one life stage to the next, i.e., from larva to nymph and from nymph to adult) and transovarial transmission (from female to offspring). Lastly, in addition to biological transmission via ticks, mechanical transmission (without pathogen reproduction within

1. This document is IN1327, one of a series of the Entomology and Nematology Department, UF/IFAS Extension. Original publication date July 2021. Visit the EDIS website at https://edis.ifas.ufl.edu for the currently supported version of this publication.

2. Yuexun Tian, graduate student, graduate assistantship supported by the CDC Southeastern Center of Excellence in Vector Borne Diseases; Cynthia C. Lord, associate professor; and Eva A. Buckner, assistant professor and state Extension specialist; Entomology and Nematology Department; UF/IFAS Florida Medical Entomology Laboratory, Vero Beach, FL 32962.

The Institute of Food and Agricultural Sciences (IFAS) is an Equal Opportunity Institution authorized to provide research, educational information and other services

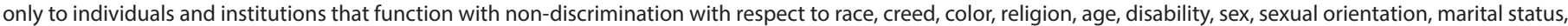

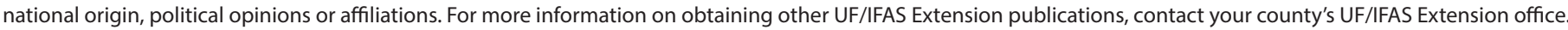
U.S. Department of Agriculture, UF/IFAS Extension Service, University of Florida, IFAS, Florida A \& M University Cooperative Extension Program, and Boards of County Commissioners Cooperating. Nick T. Place, dean for UF/IFAS Extension. 
the host) by biting flies, particularly horse flies, has been reported (Yunik et al. 2016).

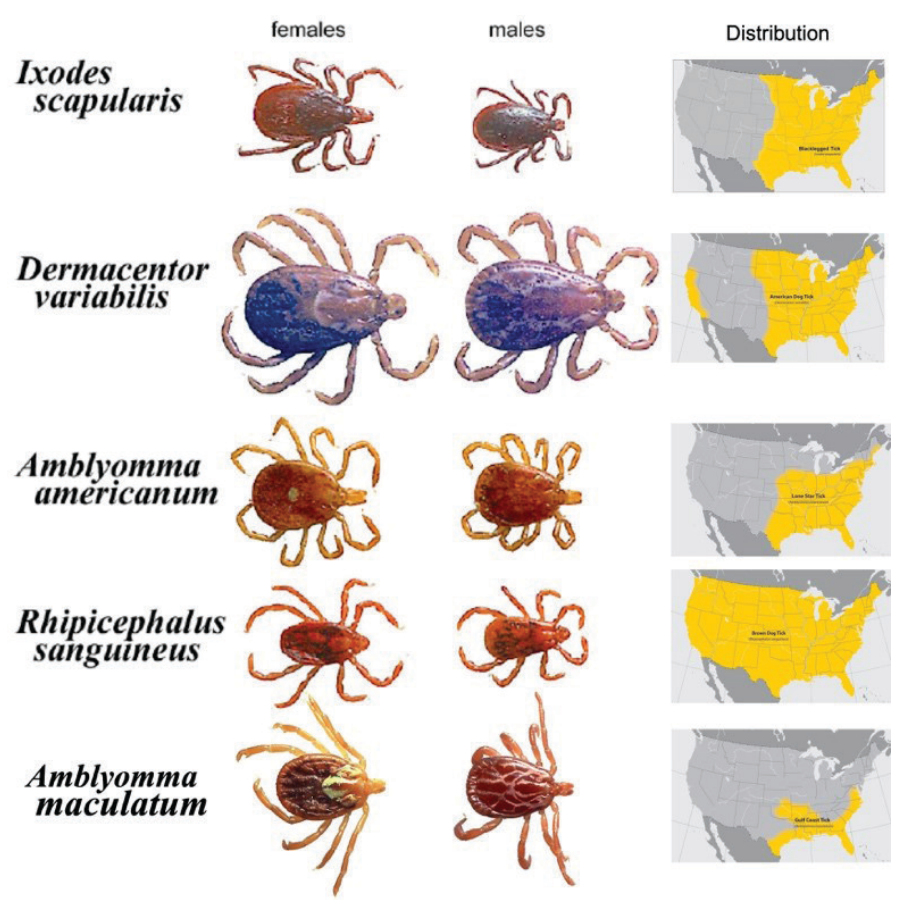

Figure 2. Female and male of Ixodes scapularis (blacklegged tick), Dermacentor variabilis (American dog tick), Amblyomma americanum (lone star tick), Rhipicephalus sanguineus (brown dog tick), and Amblyomma maculatum (Gulf Coast tick) and their geographical distributions in the United States.

Credits: James Newman, University of Florida

This publication provides an in-depth description of Ehrlichia and Anaplasma species that cause diseases in humans and/or other animals. Its intended audience is professionals in the fields of acarology, medical entomology, medicine, public health, veterinary medicine, and livestock production, as well as anyone interested in learning more about potential pathogens transmitted by ticks. One thing worth noting is that, due to an improved understanding of these organisms, the names for some Ehrlichia and Anaplasma species have been revised. Thus, you may find different names in old and new literature that are referring to the same organism.

\section{Human Disease-Causing Ehrlichia and Anaplasma in the United \\ States}

Currently in the United States, Ehrlichia chaffeensis, Ehrlichia ewingii, Ehrlichia muris eauclairensis (formerly Ehrlichia muris-like agent), and Panola Mountain Ehrlichia species (Reeves et al. 2008, CDC 2020a) as well as Anaplasma phagocytophilum (formerly known as E. equi and E. phagocytophilum), have been found to cause disease in humans (CDC 2020b). However, other species may be identified in the future as detection methods are improved. These five pathogens are transmitted transstadially in ticks but not transovarially. The early symptoms of the diseases usually start within 7-14 days after exposure, can be similar for the two diseases, and include malaise (feeling unwell), fever, chills, myalgia (muscle pain), headache, nausea, and rash. Early symptoms in ehrlichiosis cases can sometimes progress to symptoms associated with severe, late-stage illness, such as respiratory failure, uncontrolled bleeding, brain or nervous system damage, organ failure, and death. Severe illness is more likely in those with delayed antibiotic treatment or weakened immune systems and/or the elderly or very young (CDC 2020a).

Ehrlichia chaffeensis is the most common bacterium reported in human ehrlichiosis cases and was first described as a human pathogen in 1986 (Dumler et al. 2007). The lone star tick (Amblyomma americanum), which is distributed mainly in the south-central and eastern United States but is moving into the Midwest, is the main vector (Figure 2). White-tailed deer (Odocoileus virginianus) are recognized as reservoirs in the transmission cycle of Ehrlichia chaffeensis. Domestic dogs (Paddock and Childs 2003), raccoons (Ganguly and Mukhopadhayay 2008), and coyotes (Kocan et al. 2000) are also possible reservoirs. Lone star ticks can become infected with Ehrlichia chaffeensis when they feed on infected hosts (Long et al. 2003). The pathogen remains in the infected tick and can be transmitted to reservoir species or to human hosts through feeding during the subsequent tick nymphal and adult stages.

Ehrlichia chaffeensis is the causative agent responsible for human monocytic ehrlichiosis (HME), mainly by infecting monocytic cells (a type of white blood cell) as well as other cells such as lymphocytes (Ganguly and Mukhopadhayay 2008). According to CDC statistics, the number of reported HME cases has increased from 200 in the year 2000 to more than 1,799 in 2018 . However, the fatality rate has decreased. Both of these trends may be, in part, due to increased recognition and diagnosis of cases based on clinical suspicion. Also, early treatment with the antibiotic doxycycline can prevent death and severe illness (CDC 2020a). Although HME cases have been reported throughout the year, summer (June and July) is the peak period, that corresponds to the peak abundance of lone star tick nymphs (Figure 3) and adults (Figure 2) (from April to September) (Holderman and Kaufman 2013). HME is most common in the southeastern United States but human cases have been diagnosed in most US states. This is likely a 
result of human travel, considering that the vector does not occur in every state.

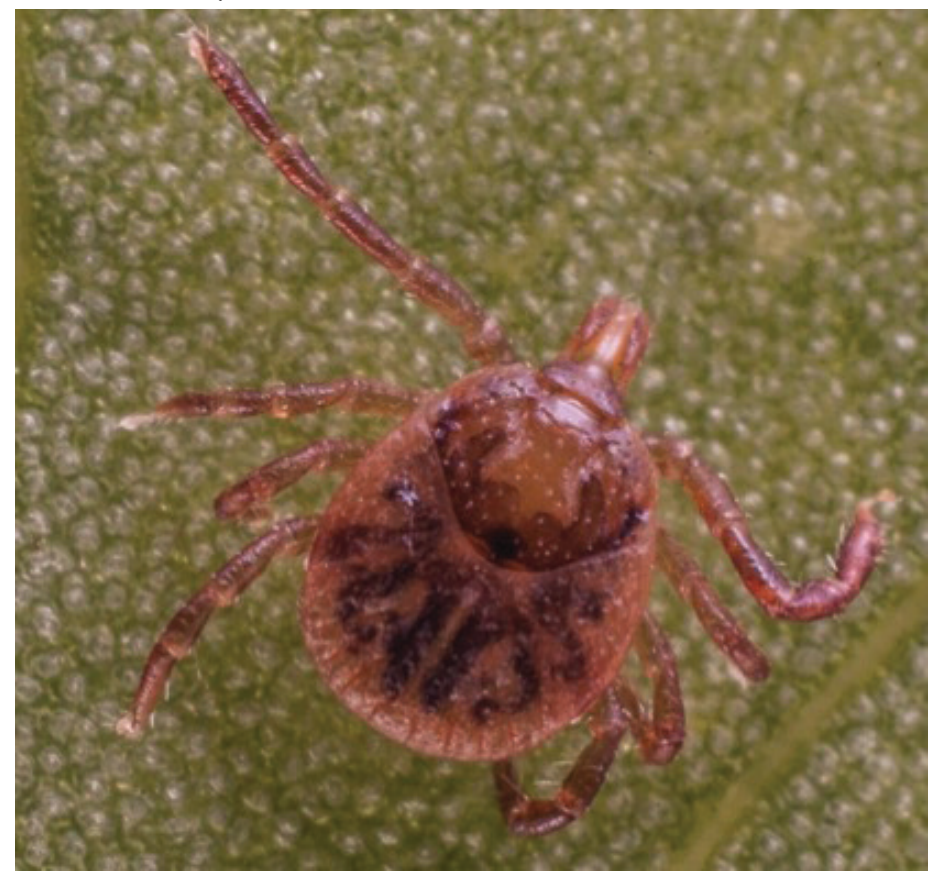

Figure 3. Lone star tick nymph.

Credits: James Newman, University of Florida

\section{Ehrlichia ewingii}

Ehrlichia ewingii, which was first described as a human pathogen in 1996 (Dumler et al. 2007), is also vectored by the lone star tick and therefore shares a distribution similar to that of Ehrlichia chaffeensis. It also displays a similar transmission cycle and human symptoms, which contributes to the difficulty in distinguishing between infections caused by the two species. These two bacteria can be distinguished by genetic methods that are used in research but not clinical diagnostic tools (based on medical tests and reported symptoms). However, the presence of a rash is rare in E. ewingii cases compared to E. chaffeensis (Heitman et al. 2016). From 2008-2018, a total of 218 cases of E. ewingii ehrlichiosis were reported to CDC, and no fatal cases of $E$. ewingii ehrlichiosis have been reported (CDC 2020a).

\section{Ehrlichia muris eauclairensis}

Ehrlichia muris eauclairensis was known formerly as Ehrlichia muris-like agent. It is a human ehrlichiosiscausing pathogen that was first identified in patients from Minnesota and Wisconsin in 2009 (Pritt et al. 2011) and is transmitted by the blacklegged tick (Ixodes scapularis) that can be found primarily in the eastern half of the United States (Figure 2). Since its first detection, more than 115 cases of ehrlichiosis caused by Ehrlichia muris eauclairensis have been identified in the upper midwestern region of the United States. No deaths have been described. The white-footed mouse plays an important role in maintaining
Ehrlichia muris eauclairensis in nature (Karpathy et al. 2016). Blacklegged ticks (Figure 4) can be infected by Ehrlichia muris eauclairensis when they feed on infected hosts or co-feed on uninfected hosts with infected ticks (Karpathy et al. 2016). However, the complete ecology of Ehrlichia muris eauclairensis is not understood at present.

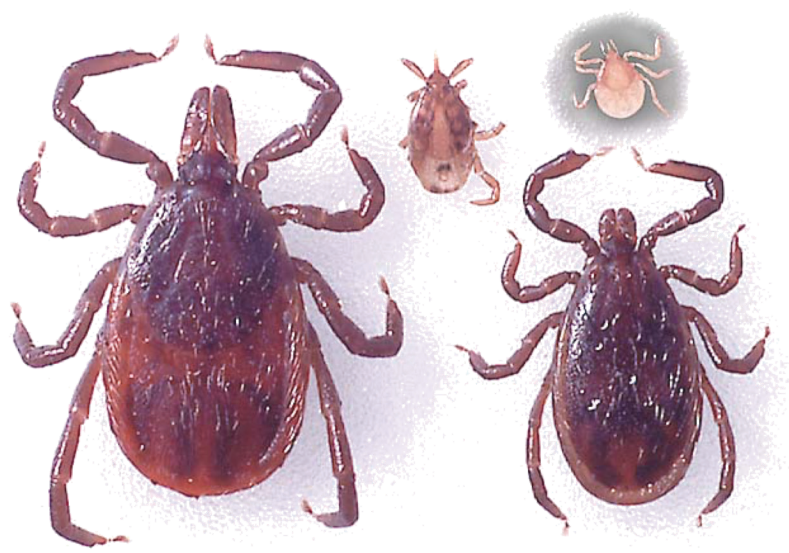

Figure 4. The three life stages of a blacklegged tick: larva, nymph, and adult female and male.

Credits: James Newman, University of Florida

\section{Panola Mountain Ehrlichia Species}

The Panola Mountain Ehrlichia species (PMES) was described as a disease-causing pathogen following its detection from a goat after being exposed to lone star tickscollected at Panola Mountain State Park, Georgia, in 2005. The goat developed a fever at 19 days after the tick fed on it (Loftis et al. 2006). The first human disease caused by PMES was reported in 2008. PMES was detected in blood from a patient after a bite from a nymphal lone star tick (Reeves et al. 2008). Cases of human infection with this pathogen are not as common as infection with the other three Ehrlichia species. Genetically, PMES is more closely related to Ehrlichia ruminantium (a bacterium that causes the disease heartwater in domestic and wild ruminantssee below) (Reeves et al. 2008). Quantitative polymerase chain reaction (PCR) assays can be used to differentiate PMES and Ehrlichia ruminantium (Sayler et al. 2016). The lone star tick is the recognized vector of PMES. However, this pathogen has been detected in wild-caught adult Gulf Coast ticks (Amblyomma maculatum Koch) (Figure 2) from the United States and tropical bont ticks (Amblyomma variegatum Fabricius) (Figure 5) from the Caribbean and Africa (Loftis et al. 2016). Diagnosis of PMES in patients can be accomplished using PCR and serological tests (Reeves et al. 2008).

Anaplasma phagocytophilum was formerly referred to as E. equi or E. phagocytophilum before taxonomic revision in 2001 (Dumler et al. 2001) and is responsible for human 
granulocytic anaplasmosis (HGA), previously known as human granulocytic ehrlichiosis (CDC 2020b). The whitefooted mouse is the major mammal reservoir of Anaplasma phagocytophilum in nature, although other small mammals, white-tailed deer, other ruminants, and birds can be infected (Dumler et al. 2005, Keesing et al. 2012). Humans acquire the pathogen through infected tick bites (blacklegged ticks and western blacklegged ticks, I. pacificus, in the United States) and begin to show symptoms within one to two weeks after the tick bite (CDC 2020b). Anaplasma phagocytophilum in vertebrates can be detected by PCR assay and serologic testing (Sanchez et al. 2016).

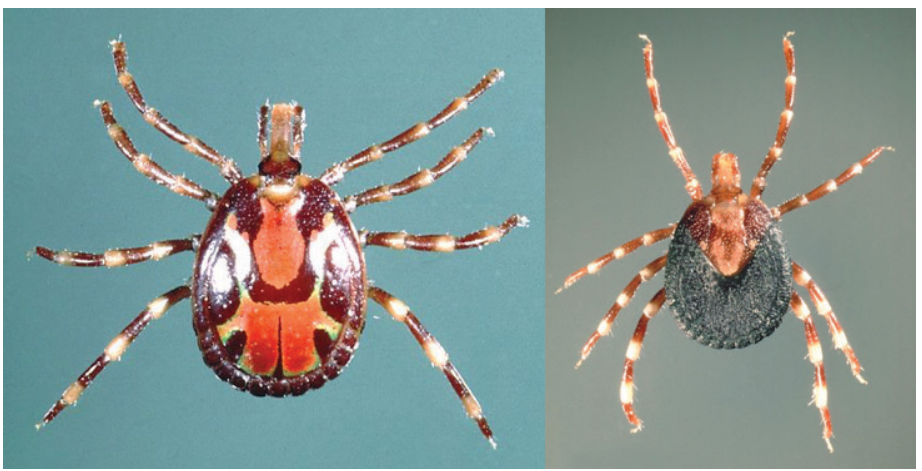

Figure 5. Male (left) and female (right) tropical bont ticks.

Credits: Alan Walker, University of Edinburgh, UK

\section{Ehrlichiosis and Anaplasmosis in Other Vertebrates}

\section{Ehrlichia canis}

Ehrlichia canis is one of the common Ehrlichia bacteria that causes ehrlichiosis in canine species. It infects mononuclear cells (types of white blood cells) and causes canine monocytic ehrlichiosis (CME) (Harrus and Waner 2011), which also is referred to as canine tropical pancytopenia (condition in which there is a reduction of red and white blood cells and platelets) due to the reduction of all blood cell types observed in this disease (Nicholson et al. 2019). CME caused by Ehrlichia canis was first identified in Algeria in 1935 and is now found worldwide. Ehrlichia canis is transmitted by the brown dog tick (Rhipicephalus sanguineus Latreille) (Figure 2) and can be maintained between tick stages and transmitted to susceptible dogs during nymph or adult blood feeding (Nicholson et al. 2019). All dog breeds can be infected with this species, but German shepherds and Siberian huskies are more susceptible to acute severe illness (Gahalot et al. 2017). Dogs infected with Ehrlichia canis usually experience high fever, low appetite, and abdominal pain (Moreira et al 2003). Some infections may progress to a chronic phase with less severe symptoms after the acute infection (Harrus and Waner 2011, Nicholson et al. 2019). Diagnosis of Ehrlichia canis can be made by examining platelet counts and through serological tests. However, co-infections with other tick-borne pathogens can affect the clinical symptoms. PCR and genetic sequencing can be used to provide a definitive confirmatory result (Harrus and Waner 2011).

\section{Ehrlichia ewingii and Ehrlichia chaffeensis}

Ehrlichia ewingii and Ehrlichia chaffeensis, transmitted by the lone star tick, are also responsible for canine ehrlichiosis. Based on serological studies of preserved canine serum samples, Ehrlichia ewingii is the most prevalent species that causes canine ehrlichiosis in North America, especially in the central and southeastern United States (Beall et al. 2012). This bacterium infects granulocytes (a granulocyte is a type of white blood cell) and causes canine granulocytic ehrlichiosis (CGE). Dogs can acquire Ehrlichia ewingii through bites from infected ticks, are able to maintain the pathogen for over two years without developing clinical signs, and serve as reservoir hosts of the pathogen (Starkey et al. 2015). White-tailed deer may serve as reservoir hosts as well (Nicholson et al. 2019). Dogs infected with Ehrlichia ewingii usually exhibit kidney disease, anemia, and increased liver enzyme activities (Qurollo et al. 2019). Ehrlichia ewingii cannot be distinguished morphologically from Anaplasma phagocytophilum in blood smears. Molecular assays must be conducted to provide accurate diagnosis (Nicholson et al. 2019). Dogs in the southcentral and southeastern United States can be infected with Ehrlichia chaffeensis. Ehrlichia chaffeensis rarely causes clinical disease in dogs by itself with more severe disease symptoms developing with other Ehrlichia species co-infections (Nicholson et al. 2019).

\section{Ehrlichia ruminantium}

Ehrlichia ruminantium, formerly known as Cowdria ruminantium, is the agent that causes heartwater disease in domestic ruminants, especially cattle, sheep, and goats, as well as some wild animals such as white-tailed deer (Peter et al. 2002). Ehrlichia ruminantium is transmitted by at least 10 Amblyomma species, with the tropical bont tick and the bont tick (Amblyomma hebraeum Koch) (Figure 6) serving as the primary vectors. Heartwater occurs mainly in sub-Saharan Africa and surrounding islands, such as Madagascar. It is also found in the Caribbean islands after its 19th century introduction through cattle imported from West Africa carrying infected ticks (Allsopp 2015, Nicholson et al. 2019). Infected animals usually develop a fever and loss of appetite, and demonstrate behavioral signs such as abnormal walking. Diarrhea has been observed in cattle. However, age, species, breed of the infected animal, and the 
Ehrlichia ruminantium genotype may alter clinical signs, making diagnosis difficult (Allsopp 2015). An animal's age, species, breed, and previous exposures also influence its disease outcome. Mortality rates in susceptible livestock can range from $<10 \%$ to $90 \%$ (Spickler 2015). Animals that survive infection acquire immunity. Examination of dead animals often reveals massive fluid accumulation in the heart, lungs, and other organs (Nicholson et al. 2019) (Figure 7). Healthy ruminant hosts can carry the pathogen and transmit infection to feeding ticks for almost one year (Allsopp 2010). Serological tests can diagnose this pathogen in ruminants, while molecular tests can identify the pathogen in both ruminants and infected ticks (Allsopp 2015). Heartwater management can be carried out using tick control, selection of resistant breeds of livestock, antibiotic treatment of infected animals, application of acaricides, and a preventative vaccination.

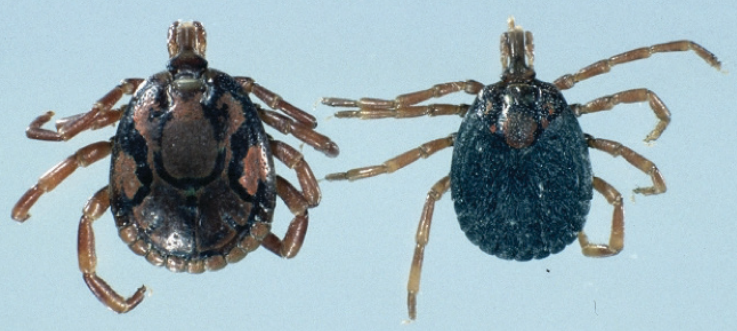

Figure 6. Male (left) and female (right) bont ticks from South Africa. Credits: James Occi. Rutgers University

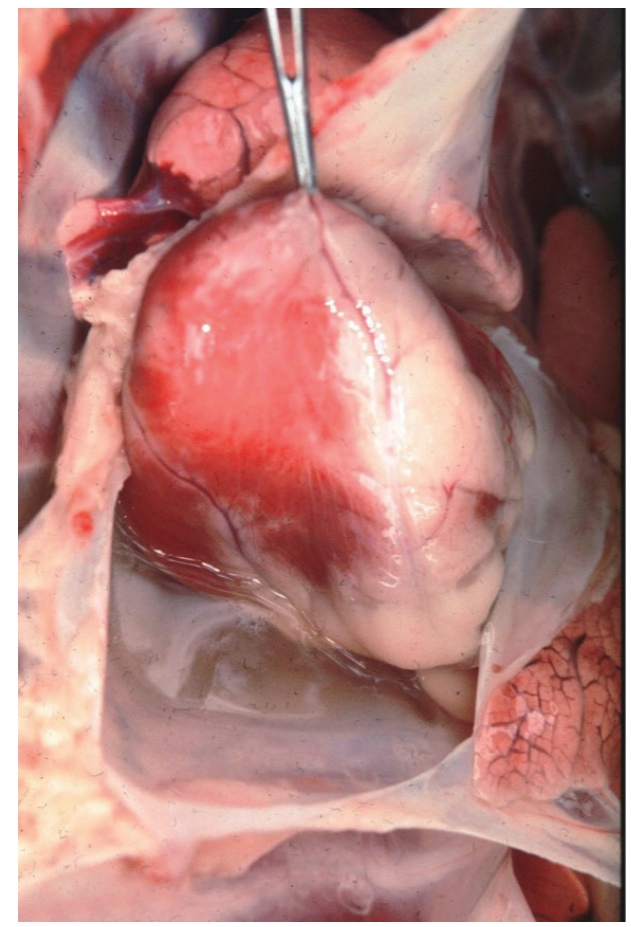

Figure 7. Heart of a goat that died of heartwater disease. Credits: Wikimedia Commons. Alan Walker, University of Edinburgh, UK
Heartwater is one of the most important diseases of livestock in Africa and has caused significant economic losses in Africa and the Caribbean due to livestock death and difficulty in introducing high-yielding livestock breeds, which are more susceptible to Ehrlichia ruminantium (Allsopp 2015). The average animal product losses caused by heartwater in southern Africa were estimated to be $\$ 48$ million annually (Minjauw et al. 2000) and \$5.6 million in Zimbabwe (Mukhebi et al. 1999). With increased animal trade in the global market, heartwater has posed a potential threat to wildlife and to the livestock industry in the United States (Introduction risk is discussed below), and the United States Department of Agriculture projects an estimated \$762 million in annual losses should a US outbreak occur.

\section{Anaplasma phagocytophilum}

Anaplasma phagocytophilum is a disease-causing pathogen in livestock and other animals (Dugat et al. 2017) in addition to humans. The vectors of Anaplasma phagocytophilum are ticks in the genus Ixodes; however, vector species vary based on geographic distribution. Vectors include: castor bean ticks (Ixodes ricinus Linnaeus) in Europe, blacklegged ticks in the eastern United States, western blacklegged ticks and Ixodes spinipalpis Hadwen and Nuttall in the western United States, and taiga ticks (Ixodes persulcatus Schulze) in Asia (Dugat et al. 2015). Anaplasma phagocytophilum can be transmitted between tick stages but not through transovarial transmission (Nicholson et al. 2019). Rodents and white-tailed deer serve as reservoir hosts in the transmission cycle (Dugat et al. 2015). The most common symptoms in ruminants are high fever $\left(>41^{\circ} \mathrm{C}\right)$, loss of appetite, and sudden milk yield reduction (dairy cattle), which causes significant economic impacts in Europe (Stuen et al. 2013). However, the severity of symptoms is affected by multiple factors including the animal's age, immunity, and species, as well as pathogen variants (Stuen et al. 2013). Anaplasma phagocytophilum can infect horses and cause equine granulocytic anaplasmosis (EGA) with symptoms of fever, weakness, anemia, and reduction of white blood cells (Dugat et al. 2015). EGA is an endemic disease, and its prevalence varies in different regions (Saleem et al. 2018). During the initial fever period, diagnosis can be accomplished by microscopy of a blood smear, while PCR and serological tests can be used during the later stages after infection (Stuen et al. 2013).

\section{Anaplasma marginale}

Anaplasma marginale infects red blood cells and causes anaplasmosis in cattle and sheep throughout most the world (Nicholson et al. 2019). Bovine anaplasmosis, caused 
by Anaplasma marginale, is considered the most important rickettsial disease in cattle and results in significant economic loss in livestock production (Quiroz-Castañeda et al. 2016).

This pathogen can be transmitted biologically by approximately 20 hard tick species. Rocky Mountain wood ticks (Dermacentor andersoni Stiles) (Figure 8) and American dog ticks serve as primary vectors in the United States (Kocan et al. 2010). In particular, male Rocky Mountain wood ticks are important vectors due to multiple-host feeding, the shorter extrinsic incubation period (pathogen cycle in the tick), and shorter feeding periods on hosts compared to female ticks (Nicholson et al. 2019). For American dog ticks, white-tailed deer are an important host for these tick species and can be infected, but are not a competent reservoir of Anaplasma marginale, which means uninfected ticks cannot acquire this pathogen from white-tailed deer. Mule deer serve as primary hosts and competent reservoirs in the western United States (Nicholson et al. 2019).

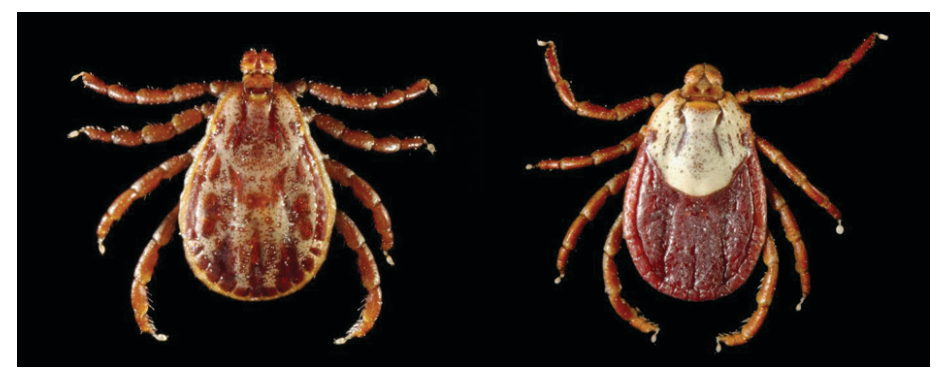

Figure 8. Male (left) and female (right) Rocky Mountain wood ticks. Credits: Centers for Disease Control and Prevention

Blood-sucking flies, such as the stable fly (Stomoxys calcitrans Linnaeus) and Tabanidae species (such as the horse fly and the deer fly), and blood-contaminated fomites, such as re-used needles and ear tag application tools, can also serve as mechanical transmission vectors of Anaplasma marginale (Kocan et al. 2010). While mechanical transmission may not be as effective as biological transmission via ticks (Scoles et al. 2005), mechanical transmission seems to be the primary route when Anaplasma marginale strains (a genetic variant or subtype of the bacteria) are not infective for ticks and in areas without tick populations (Kocan et al. 2004).

The severity of symptoms in infected animals increases with age. Without adequate treatments, recovered animals maintain low-level parasitemia and develop lifelong immunity to clinical disease (Eriks et al. 1989). However, they can serve as reservoirs by providing infective blood sources for both mechanical and biological transmission (Kocan et al. 2003). Diagnosis of bovine anaplasmosis may be made based on animal location, season, and clinical signs, but requires laboratory tests, such as microscopic evaluation of stained blood smears and serological tests, to confirm the infection (Kocan et al. 2010).

\section{Florida Situation}

Several tick species that transmit Ehrlichia or Anaplasma bacteria are present in Florida, including brown dog ticks, American dog ticks, lone star ticks, Gulf Coast ticks, and blacklegged ticks. Human ehrlichiosis and anaplasmosis have not been a severe problem in Florida with no more than 40 confirmed cases every year for each disease (FDOH 2018). However, underreporting or misdiagnosis of cases may occur due to mild symptoms and lack of laboratory testing.

In 2018, there were 4,899 reported positive canine ehrlichiosis cases identified from 273,051 tested samples and 1,410 canine anaplasmosis cases identified from 273,001 tested samples (CAPC 2020). For more information, please visit the Companion Animal Parasite Council's website. Infected ticks may be brought to Florida by migratory birds or on imported reptiles, which could result in the introduction of Ehrlichia ruminantium into our native tick species, such as Gulf Coast ticks, which have been demonstrated to be competent vectors of Ehrlichia ruminantium in the laboratory (Mahan et al. 2000). A breeding population of African tortoise ticks (Amblyomma marmoreum Koch), reported to be a competent vector of Ehrlichia ruminantium within the laboratory (Peter et al. 2000), was discovered in a reptile breeding facility located in Florida (Allan et al. 1998). It was thought to be introduced with the tortoises imported from Africa. This tick population was eradicated, but this example shows the potential for introduction. Other exotic tick species, such as central African tortoise tick (Amblyomma sparsum Neumann), have been found on imported reptiles in Florida (Burridge et al. 2000). Thus, reptile importation is closely monitored in Florida.

\section{Prevention and Management}

For human ehrlichiosis and anaplasmosis, there is no vaccine that can be used, and the best prevention is tick management. Ticks usually live in grassy, brushy, or wooded areas, except the brown dog tick that can survive both indoors and outdoors. Thus, many people acquire ticks from their yard or from hiking. There are multiple personal protection methods that can be used to reduce human-tick contact and avoid tick bites, such as long pants tucked into socks, acaricide-treated clothes, Environmental Protection Agency-registered insect repellents (for more information, please visit EPA), and conducting thorough body checks for ticks after visiting tick habitat. For other animals, 
tick prevention can be accomplished by many methods including sprays, spot-ons, collars, and ear tags containing approved acaricides such as permethrin, fipronil, amitraz, as well as tick checks and grooming. However, all acaricide uses must follow label directions to avoid poisoning and acaricide resistance in tick populations. Consult with your veterinarian because some species or breeds are sensitive to some acaricides, and severe illness or death can result. Some products are available only by prescription.

Ticks found on humans and other animals should be removed immediately using fine-tipped forceps to reduce the risk of pathogen infections. When removing the ticks, grasp the tick as near the skin as possible and pull straight back without twisting to avoid breaking and leaving the mouthparts in the skin, which can cause bacterial infections (Figure 9). After tick removal, symptoms should be monitored closely. If illness occurs within 30 days of a tick bite, a doctor or veterinarian visit should be made. Only a doctor or a veterinarian can prescribe the appropriate treatments for ehrlichiosis and anaplasmosis.
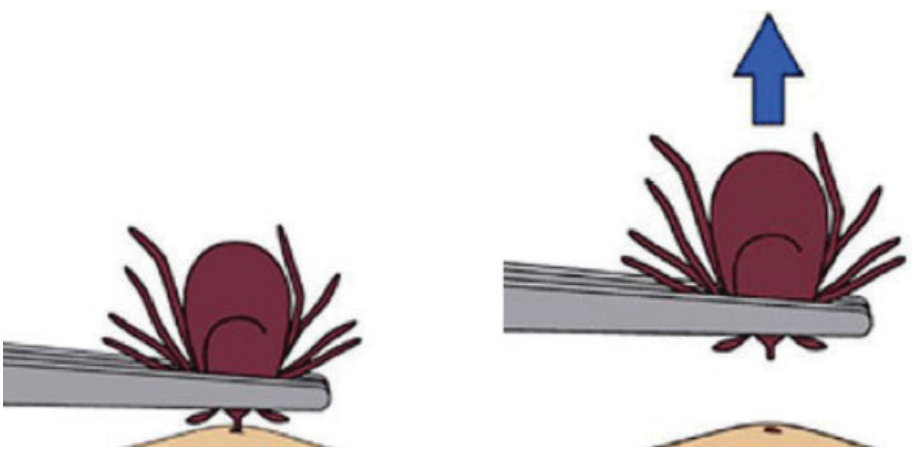

Figure 9. How to properly remove a tick using tweezers. Credits: Centers for Disease Control and Prevention

For more information, please visit Ticks (Family Ixoididae), EDIS Publication \#ENY-206, the CDC's Ehrlichiosis website, and the CDC's Anaplasmosis website.

\section{References}

Allan, S. A., L. A. Simmons, and M. J. Burridge. 1998. "Establishment of the Tortoise Tick Amblyomma marmoreum (Acari : Ixodidae) on a Reptile-Breeding Facility in Florida." Journal of Medical Entomology 35:621-624. DOI: 10.1093/jmedent/35.5.621.

Allsopp, B. A. 2010. "Natural History of Ehrlichia ruminantium." Veterinary Parasitology 167:123-135. DOI: 10.1016/j. vetpar.2009.09.014.

Allsopp, B. A. 2015. "Heartwater-Ehrlichia ruminantium Infection." Revue Scientifique et Technique OIE 34:557-568. DOI: 10.20506/rst.34.2.2379.
Beall, M. J., A. R. Alleman, E. B. Breitschwerdt, L. A. Cohn, C. G. Couto, M. W. Dryden, L. C. Guptill, et al. 2012.

"Seroprevalence of Ehrlichia canis, Ehrlichia chaffeensis and Ehrlichia ewingii in Dogs in North America." Parasite Vector 5. DOI: 10.1186/1756-3305-5-29.

Burridge, M. J., L. A. Simmons, and S. A. Allan. 2000. "Introduction of Potential Heartwater Vectors and Other Exotic Ticks into Florida on Imported Reptiles." Journal of Parasitology 86:700-704. DOI: 10.1645/0022-3395(2000)086[0700:Iophva]2.0.Co;2.

CAPC (Companion Animal Parasite Council). 2020. https://capcvet.org/maps/\#2020/all/anaplasmosis/dog/ united-states/ (Last accessed: August 14, 2020).

CDC (Centers of Disease Control and Prevention). 2020a. "Ehrlichiosis." https://www.cdc.gov/ehrlichiosis/index.html (Last accessed: August 14, 2020).

CDC (Centers of Disease Control and Prevention). 2020b. "Anaplasmosis." https://www.cdc.gov/anaplasmosis/stats/ index.html (Last accessed: August 14, 2020).

Dugat, T., A. Leblond, N. Keck, R. Maillard, H. Boulouis, and N. Haddad. 2015. "Opening the Black Box of Anaplasma phagocytophilum Diversity: Current Situation and Future Perspectives." Frontiers in Cellular and Infection Microbiology 5. DOI: 10.3389/fcimb.2015.00061.

Dugat, T., A. Leblond, N. Keck, A. C. Lagree, I. Desjardins, A. Joulie, S. Pradier, B. Durand, H. J. Boulouis, and N. Haddad. 2017. "One Particular Anaplasma phagocytophilum Ecotype Infects Cattle in the Camargue, France." Parasite Vectors 10:371. DOI: 10.1186/s13071-017-2305-3.

Dumler, J. S., F. B. Anthony, C. P. J. Bekke, G. A. Dasch, G. H. Palmer, S. C. Ray, Y. Rikihisa, and F. R. Rurangirwa. 2001. "Reorganization of Genera in the Families Rickettsiaceae and Anaplasmataceae in the Order Rickettsiales: Unification of Some Species of Ehrlichia with Anaplasma, Cowdria with Ehrlichia and Ehrlichia with Neorickettsia, Descriptions of Six New Species Combinations and Designation of Ehrlichia equi and 'HGE agent' as Subjective Synonyms of Ehrlichia phagocytophila." International Journal of Systematic and Evolutionary Microbiology 51:2145-2165. DOI: 10.1099/00207713-51-6-2145.

Dumler, J. S., J. E. Madigan, N. Pusterla, and J. S. Bakken. 2007. "Ehrlichioses in Humans: Epidemiology, Clinical Presentation, Diagnosis, and Treatment." Clinical Infectious Diseases 45 Suppl 1: S45-51. DOI: 10.1086/518146. 
Dumler, J. S., K. S. Choi, J. C. Garcia-Garcia, N. S. Barat, D. G. Scorpio, J. W. Garyu, D. J. Grab, and J. S. Bakken. 2005. "Human Granulocytic Anaplasmosis and Anaplasma phagocytophilum." Emerging Infectious Diseases 11: 1828-1834. DOI: 10.3201/eid1112.050898.

Eriks, I. S., G. H. Palmer, T. C. McGuire, D. R. Allred, A. F. Barbet. 1989. "Detection and Quantitation of Anaplasma marginale in Carrier Cattle by Using a Nucleic Acid Probe." Journal of Clinical Microbiology 27: 279-284. DOI: 10.1128/ Jcm.27.2.279-284.1989.

FDOH (Florida Department of Health.) 2018. FLHealthCharts. http://www.flhealthcharts.com/charts/Default.aspx (Last accessed: August 25, 2020).

Gahalot, S., D. Meena, R. Singh, Y. Singh, M. Mali, J. Bargurjar, N. Jeph, and S. Meena. 2017. "Therapeutic Management of Canine Ehrlichiosis in a German Shepherd Bitch: A Case Report." Research Journal for Veterinary Practitioners 5:37-39. DOI: 10.17582/journal.rjvp/2017/5.4.37.39.

Ganguly, S., and S. K. Mukhopadhayay. 2008. "Tick-Borne Ehrlichiosis Infection in Human Beings." Journal of Vector Borne Diseases 45:273-280. PMID: 19248653.

Harrus, S., and T. Waner. 2011. "Diagnosis of Canine Monocytotropic Ehrlichiosis (Ehrlichia canis): An Overview." Veterinary Journal 187:292-296. DOI: 10.1016/j. tvjl.2010.02.001.

Heitman, K. N., F. S. Dahlgren, N. A. Drexler, R. F. Massung, and C. B. Behravesh. 2016. "Increasing Incidence of Ehrlichiosis in the United States: A Summary of National Surveillance of Ehrlichia chaffeensis and Ehrlichia ewingii Infections in the United States, 2008-2012." The American Journal of Tropical Medicine and Hygiene 94:52-60. DOI: 10.4269/ajtmh.15-0540.

Holderman, C. J., and P. E. Kaufman. 2013. "Lone Star Tick-Amblyomma americanum (Linnaeus).” http:// entnemdept.ufl.edu/creatures/urban/medical/lone_star_ tick.htm (Last accessed: August 14, 2020).

Karpathy, S. E., M. E. Allerdice, M. Sheth, G. A. Dasch, and M. L. Levin. 2016. "Co-Feeding Transmission of the Ehrlichia muris-like Agent to Mice (Mus musculus)." VectorBorne and Zoonotic Diseases 16:145-150. DOI: 10.1089/ vbz.2015.1878.
Keesing, F., M. H. Hersh, M. Tibbetts, D. J. McHenry, S. Duerr, J. Brunner, M. Killilea, K. LoGiudice, K. A. Schmidt, and R. S. Ostfeld. 2012. "Reservoir Competence of Vertebrate Hosts for Anaplasma phagocytophilum." Emerging Infectious Diseases 18:2013-2016. DOI: 10.3201/ eid1812.120919.

Kocan, A. A., G. C. Levesque, L. C. Whitworth, G. L. Murphy, S. A. Ewing, and R. W. Barker. 2000. "Naturally Occurring Ehrlichia chaffeensis Infection in Coyotes from Oklahoma." Emerging Infectious Diseases 6:477-480. DOI: 10.3201/eid0605.000505.

Kocan, K. M., J. de la Fuente, A. A. Guglielmone, and R. D. Melendez. 2003. "Antigens and Alternatives for Control of Anaplasma marginale Infection in Cattle." Clinical Microbiology Reviews 16:698-712. DOI: 10.1128/ Cmr.16.4.698-712.2003.

Kocan, K. M., J. de la Fuente, E. F. Blouin, J. F. Coetzee, and S. A. Ewing. 2010. "The Natural History of Anaplasma marginale." Veternary Parasitology 167:95-107. DOI: 10.1016/j.vetpar.2009.09.012.

Loftis, A. D., W. K. Reeves, J. P. Spurlock, S. M. Mahan, D. R. Troughton, G. A. Dasch, and M. L. Levin. 2006. "Infection of a Goat with a Tick-Transmitted Ehrlichia from Georgia, U.S.A., That Is Closely Related to Ehrlichia ruminantium." Journal of Vector Ecology 31:213-223. DOI: 10.3376/1081-1710(2006)31[213:ioagwa]2.0.co;2.

Loftis, A. D., P. J. Kelly, C. D. Paddock, K. Blount, J. W. Johnson, E. R. Gleim, M. J. Yabsley, M. L. Levin, and L. Beati. 2016. "Panola Mountain Ehrlichia in Amblyomma maculatum from the United States and Amblyomma variegatum (Acari: Ixodidae) from the Caribbean and Africa." Journal of Medical Entomology 53:696-698. DOI: 10.1093/jme/tjv240.

Long, S. W., X. F. Zhang, J. Z. Zhang, R. P. Ruble, P. Teel, and X. J. Yu. 2003. "Evaluation of Transovarial Transmission and Transmissibility of Ehrlichia chaffeensis (Rickettsiales: Anaplasmataceae) in Amblyomma americanum (Acari : Ixodidae)." Journal of Medical Entomology 40: 1000-1004. DOI: 10.1603/0022-2585-40.6.1000.

Mahan, S. M., T. F. Peter, B. H. Simbi, K. Kocan, E. Camus, A. F. Barbet, and M. J. Burridge. 2000. "Comparison of Efficacy of American and African Amblyomma Ticks as Vectors of Heartwater (Cowdria ruminantium) Infection by Molecular Analyses and Transmission Trials." Journal of Parasitology 86:44-49. DOI: 10.2307/3284906. 
Minjauw, B., R. Kruska, A. Odero, T. F. Randolph, J. J. McDermott, S. Mahan, and B. D. Perry. 2000. "Economic Impact Assessment of Cowdria ruminantium Infection and Its Control in Southern Africa." Proceedings of the Ninth International Symposium on Veterinary Epidemiology and Economics, Breckenridge, CO, Paper 645

Moreira, S. M., C. V. Bastos, R. B. Araujo, M. Santos, and L. M. F. Passos. 2003. "Retrospective Study (1998-2001) on Canine Ehrlichiosis in Belo Horizonte, MG, Brazil." Arquivo Brasileiro De Medicina Veterinaria E Zootecnia 55:141-147. DOI: 10.1590/S0102-09352003000200003.

Mukhebi, A. W., T. Chamboko, C. J. O'Callaghan, T. F. Peter, R. L. Kruska, G. F. Medley, S. M. Mahan, and B. D. Perry. 1999. "An Assessment of the Economic Impact of Heartwater (Cowdria ruminantium Infection) and Its Control in Zimbabwe." Preventive Veterinary Medicine 39:173-189. DOI: 10.1016/s0167-5877(98)00143-3.

Nicholson, W. L., D. E. Sonenshine, B. H. Noden, R. N. Brown. 2019. “Ticks (Ixodida).” pp. 603-672. In Medical and Veterinary Entomology. $3^{\text {rd }}$ edition. Edited by R. M. Gary and L. A. Durden (ed.), Academic Press. DOI: 10.4269/ajtmh.15-0540.

Paddock, C. D., and J. E. Childs. 2003. "Ehrlichia chaffeensis: A Prototypical Emerging Pathogen." Clinical Microbiology Reviews 16:37-64. DOI: 10.1128/cmr.16.1.37-64.2003.

Peter, T. F., M. J. Burridge, and S. M. Mahan. 2000. "Competence of the African Tortoise Tick, Amblyomma marmoreum (Acari : Ixodidae), as a Vector of the Agent of Heartwater (Cowdria ruminantium)." Journal of Parasitology 86:438-441. DOI: $10.2307 / 3284854$.

Peter, T. F., M. J. Burridge, and S. M. Mahan. 2002. "Ehrlichia ruminantium Infection (Heartwater) in Wild Animals." Trends in Parasitology 18:214-218. DOI: 10.1016/ S1471-4922(02)02251-1.

Pritt, B. S., L. M. Sloan, D. K. H. Johnson, U. G. Munderloh, S. M. Paskewitz, K. M. McElroy, J. D. McFadden, et al. 2011. "Emergence of a New Pathogenic Ehrlichia Species, Wisconsin and Minnesota, 2009." New England Journal of Medicine 365:422-429. DOI: 10.1056/NEJMoa1010493.
Quiroz-Castaneda, R. E., I. Amaro-Estrada, and S. D. Rodriguez-Camarillo. 2016. "Anaplasma marginale: Diversity, Virulence, and Vaccine Landscape through a Genomics Approach.” BioMed Research International 2016. DOI: $10.1155 / 2016 / 9032085$.

Qurollo, B. A., J. Buch, R. Chandrashekar, M. J. Beall, E. B. Breitschwerdt, C. B. Yancey, A. H. Caudill, and A. Comyn. 2019. "Clinicopathological Findings in 41 Dogs (2008-2018) Naturally Infected with Ehrlichia ewingii." Journal of Veterinary Internal Medicine 33:618-629. DOI: 10.1111/jvim.15354.

Reeves, W. K., A. D. Loftis, W. L. Nicholson, and A. G. Czarkowski. 2008. "The First Report of Human Illness Associated with the Panola Mountain Ehrlichia Species: A Case Report." Journal of Medical Case Reports 2:139-141. DOI: 10.1186/1752-1947-2-139.

Sanchez, E., E. Vannier, G. P. Wormser, and L. T. Hu. 2016. "Diagnosis, Treatment, and Prevention of Lyme Disease, Human Granulocytic Anaplasmosis, and Babesiosis: A Review. Journal of the American Medical Association 315:1767-1777. DOI: 10.1001/jama.2016.2884.

Sayler, K. A., A. D. Loftis, S. M. Mahan, and A. F. Barbet. 2016. "Development of a Quantitative PCR Assay for Differentiating the Agent of Heartwater Disease, Ehrlichia ruminantium, from the Panola Mountain Ehrlichia." Transboundary and Emerging Diseases 63:E260-E269. DOI: 10.1111/tbed.12339.

Scoles, G. A., A. B. Broce, T. J. Lysyk, and G. H. Palmer. 2005. "Relative Efficiency of Biological Transmission of Anaplasma marginale (Rickettsiales: Anaplasmataceae) by Dermacentor andersoni (Acari: Ixodidae) Compared with Mechanical Transmission by Stomoxys calcitrans (Diptera : Muscidae)." Journal of Medical Entomology 42:668-675. DOI: 10.1603/0022-2585(2005)042 [0668:Reobto]2.0.Co;2.

Saleema, S., M. Ijaza, S. H. Farooqia, A. Ghaffara, A. Alia, K. Iqbala, K. Mehmoodb, H. Zhang. 2018. "Equine Granulocytic Anaplasmosis 28 Years Later." Microbial Pathogenesis 119:1-8. DOI: 10.1016/j.micpath.2018.04.001.

Spickler, A. R. 2015. "Heartwater." http://www.cfsph.iastate. edu/Factsheets/pdfs/heartwater.pdf. (Last accessed: August 14, 2020). 
Starkey, L. A., A. W. Barrett, M. J. Beall, R. Chandrashekar, B. Thatcher, P. Tyrrell, and S. E. Little. 2015. "Persistent Ehrlichia ewingii Infection in Dogs after Natural Tick Infestation." Journal of Veterinary Internal Medicine 29:552-555. DOI: $10.1111 /$ jvim. 12567.

Stuen, S., E. G. Granquist, and C. Silaghi. 2013. “Anaplasma phagocytophilum-A Widespread Multi-Host Pathogen with Highly Adaptive Strategies." Frontiers in Cellular and Infection Microbiology 3. DOI: 10.3389/fcimb.2013.00031. DOI: $10.3389 /$ fcimb.2013.00031.

Yunik, M. E., T. D. Galloway, and L. R. Lindsay. 2016. "Active Surveillance of Anaplasma marginale in Populations of Arthropod Vectors (Acari: Ixodidae; Diptera: Tabanidae) during and after an Outbreak of Bovine Anaplasmosis in Southern Manitoba, Canada." Canadian Journal of Veterinary Research 80:171-174. PMID: 27127345. 